\title{
MEAN-PERIODIC FUNCTIONS
}

\section{CARLOS A. BERENSTEIN}

Department of Mathematics

University of Maryland

College Park, Maryland 20742

U.S.A.

\section{B.A. TAYLOR}

Department of Mathematics

University of Michigan

Ann Arbor, Michigan 48109

U.S.A.

(Peceived September 25, 1979)

ABSTRACT: We show that any mean-periodic function $f$ can be represented in terms of exponential-polynomial solutions of the same convolution equation f satisfies, i.e., $u * f=0 \quad\left(\mu \in E^{\prime}\left(\mathbb{R}^{n}\right)\right)$. This extends to $n$-variables the work of L. Schwartz on mean-periodicity and also extends L. Ehrenpreis' work on partial differential equations with constant coefficients to arbitrary convolutors. We also answer a number of open questions about mean-periodic functions of one variable. The basic ingredient is our work on interpolation by entire functions in one and several complex variables. 
KEY WOROS ANO PHRASES. Mean-periodic junctions, Interpolation by chtire junctions. 1980 MATHEIATICS SUBJECT CLASSIFICATION CODES. Primary +2A84, Secondary 3902, $+102,+202,+602$.

1. The theory of mean-periodic functions, initiated by Jean Delsarte in 1935 is one of the latest, and most important, incarnations of the old subject of convolution equations, an area which in one guise or another has been a central object of interest to mathematicians over the past two hundred years. It is a subject which touches many different branches of mathematics, ranging from number theory to applied mathematics. In recent years, major contributions to the theory have been made by L. Schwartz, J.-P. Kahane, L. Ehrenpreis, and B. Malgrange, among others. The present survey is an attempt to place some of these contributions in mathematical and historical perspective and to give applications of our recent work $[7,8]$ on interpolation by entire functions which answers a number of old questions and (as usual) raises new ones. While only a few proofs have been given, we have tried to provide complete references or, at least, sources for references.

The particular aspect of the theory of mean-periodic functions we are concerned with in this paper is their representation as sums or integrals of exponentials. The main new result is the existence of such a representation for functions of more than one variable (Theorems 7,3 ). Other results that are new even for functions of one variable are the estimates of coefficients in exapansions of mean-periodic functions (Theorems $4,7,11$ ) and applications (Theorem 5 and Section 4), and a new "summability method" for summing formal expansions of meanperiodic functions which, in contrast to the original method of L. Schwartz [64], applies in any number of variables. We also mention some applications of our work on interpolation from varieties of codimension greater than 1 to certain systems of equations (Theorem 9). 
2. We begin by discussing some familiar convolution equations. Consider first a homogeneous ordinary differential equation with constant coefficients,

$$
\sum_{j=0}^{m} a_{j} D^{j} f(x)=0
$$

where $D=\frac{1}{i} \frac{d}{d x}, \quad a_{j} \in \mathbb{C}$. Euler was the first to consider the problem of finding a 22 solutions to this equation. In [25] he showed that they are given by finite sums of the form

$$
f(x)=\sum_{a} c_{a}(x) e^{-i a x}
$$

where the summation extends over all roots $\alpha$ of the algebraic equation

$$
\sum a_{j}(-\alpha)^{j}=0
$$

and the $c_{\alpha}$ are polynomials of degree $<m_{\alpha}=$ multiplicity of $a$.

Euler also studied periodic functions and proposed to write them in terms of exponentials. A periodic function (of period $2 \pi$, say) satisfies the equation

$$
f(x+\pi)-f(x-\pi)=0
$$

and is represented by an infinite series

$$
f(x)=\sum_{-\infty}^{+\infty} c_{\alpha} e^{-i a x}
$$

where the frequencies a satisfy the equation

$$
e^{i \pi a}-e^{-i \pi a}=0
$$

For the history of the theory of these series, the Fourier series, see [28, 
78]. For the theory there is the comprehensive treatise [77]. The main points we wish to recall may be summarized as follows.

THEOREM 1: (i) The Fourier series (3) of a smooth $\left(C^{\infty}\right)$ periodic function $f$ converges to $f$. Further, the series can be differentiated term by term any number of times and the resulting series converges to the corresponding derivative of $\mathrm{f}$.

(ii) The coefficients $c_{\alpha}$ of the above series satisfy the estimates

$$
c_{\alpha}=O\left(|\alpha|^{-N}\right) \text { as }|\alpha| \rightarrow \infty
$$

(iii) Conversely, any series (3) whose coefficients $c_{\alpha}$ satisfy (4) is the series of some smooth periodic function.

(iv) The coefficients are uniquely determined by $f$.

Note that these four properties are also satisfied by any finite sum (2). Observe further that (iv) can be strengthened to the following. If $f$ has also the period $2 \mathrm{p}$ (in addition to being periodic of period $2 \pi$ ) the only a for which $c_{\alpha} \neq 0$ are those for which $e^{-i \alpha x}$ is also periodic of period $2 p$. In particular, if $p$ is not commensurable with $\pi$ we have $f \equiv$ const. This uniqueness property is easily seen to be a consequence of the convergence of the series (3) in $E$.

The periodic functions and the solutions of constant coefficient differential equations are examples of mean-periodic functions, a notion introduced by Delsarte and Schwartz $[15,64]$. Following the notation of Schwartz [63], let $E=E\left(\mathbb{R}^{\mathrm{n}}\right)$ denote the space of all infinitely differentiable functions on $\mathbb{R}^{\mathrm{n}}$, endowed with the usual topology of uniform convergence of the functions and their derivatives of all orders on compact subsets of $\mathbb{R}^{\mathrm{n}}$. Recall that if $\mu$ is a distribution with compact support in $\mathbb{R}^{n}$ (i.e., $\mu \in E^{\prime}=E^{\prime}\left(\mathbb{R}^{n}\right)$, the topological dual 
space of all continuous linear functionals on $E$ ) then the convolution of $\mu$ with $\mathrm{f} \in E\left(\mathbb{R}^{\mathrm{n}}\right.$ ) is defined (formally) by

$$
(\mu+f)(x)=\int f(x-t) \mu(t) d t
$$

and precisely, by

$$
(\mu * f)(x)=\mu\left(\check{f}_{x}\right)=<\mu, \check{f}_{x}>
$$

where $f_{x}(t)=f(t-x)$ denotes the translate of $f$ by $x, \check{f}(t)=f(-t)$, and $<\mu, f=\mu(f)$ is the duality pairing of $E^{\prime}$ with $E$. One importance of the convolution product resides in the fact that the most general linear continuous operator $\mathrm{N}: E \rightarrow E$ which commutes with translations can be represented by a convolution, i.e., if $M_{x}(f(x+t))=(M f)(x+t)$ then $M f(x)=(\mu \neq f)(x)$ for some (unique).$\downarrow \in E^{\prime}$.

DEFINITION. A function $f \in E$ is called mean-periocic if there is a nonzero distribution $\mu \in E^{\prime}$ such that $\mu * \dot{\mathrm{f}}=0$. If we want to emphasize the equation satisfied by $f$ we will say that $f$ is mean-periodic with respect to $\mu$. The equation defining periodic functions is of the form

$$
(\mu * f)(x)=f(x+\pi)-f(x-\pi)=0
$$

with $H=5_{\pi}-\delta_{-\pi}$, where $\delta_{a}$ denotes the Dirac point mass measure at a. Similarly, if $5_{a}^{(j)}$ is the $j-$ th derivative of $\delta_{a}, \delta_{a}^{(j)}(g)=(-i){ }_{g}^{(j)}(a)$, and if $l=\sum_{j=0}^{m} a_{j} i_{s}{ }_{a}(j)$, then $(\mu * f)(x)=0$ is the homogeneous differential equation (1). The frequencies a which appear in the expansions (2) and (3) are the zeros of the entire holomorphic function,

$$
\hat{u}(z)=\int e^{i x \cdot z} d \mu(x)=-\mu_{x}, e^{-i x \cdot z}>
$$

which is the oring transform of $\mu$. The examples suggest that one should be 
able to expand a mean-periodic function $f$ in terms of the simplest solutions to the convolution equation it satisfies, namely the monomial-exponentials $x^{k} e^{-i a x}$ having frequencies determined by the equation

$$
\mu * e^{i \sim x}=\hat{\mu}(a) e^{i a x}=0
$$

and $\mathrm{k}-\mathrm{m}_{\mathrm{a}}=$ multiplicity of the root $\sigma$. That is, we expect that $\mu * \check{\mathrm{f}}=0$ will lead to a series

$$
f(x)=\sum_{\hat{\mu}(a)=0} c_{\alpha}(x) e^{-i a x},
$$

where the $c_{c}$ are polynomials with the same restrictions in their degree imposed above. Before drawing any conclusion we consider another example.

A particular kind of convolution equation which includes the two cases studied by Euler are the difference-differential equations. These were first considered by Condorcet in 1771 who found the exponentials with frequencies solving $\hat{\mu}(a)=0$ as particular solutions to $\mu * \check{f}=0$ (see [44, vol. 3, ch. 8] for the details). In this case the convolutor $\mu$ has as Fourier transform an exponentialpolynomial.

$$
\hat{\mu}(z)=\sum_{j=1}^{m} a_{j}(z) e^{-i \omega_{j} z},
$$

where the $\omega_{j} \in \mathbb{R}$. (The $a_{j}$ are polynomials with complex coefficients.) In the 19 th century, Cauchy and Poisson among others worked on this kind of problem (see $[44,58])$, but the first significant attempts to prove that all solutions of $\mu * \check{\mathrm{f}}=0$ are of the form (5) when $u$ represents a difference-differential operator were those of Schmidt [61], Polossuchin [60], Schürer [62] and Hilb [34]. The difficulty is that the series (5) is in general not pointwise convergent as shown by Leont'ev [47]. His example is the following. Let $\ell \in \mathbb{R}, 1 \neq 0$, and 
define the difference operator $\mu$ by

$$
\hat{\mu}(z)=4 \sin \pi z \sin \frac{\pi}{\lambda} z \text {, }
$$

explicit1y,

$$
\mu * \check{f}(x)=f\left(\frac{\pi}{\lambda}-\pi-x\right)+f\left(\pi-\frac{\pi}{\lambda}-x\right)-f\left(\pi+\frac{\pi}{\lambda}-x\right)-f\left(-\pi-\frac{\pi}{\lambda}-x\right) .
$$

If $\lambda \notin \mathbb{Q}$, the rational numbers, then all the roots of $\hat{\mu}(\alpha)=0$ are of the form $p \in \mathbf{Z}$ and $q \lambda(q \in \mathbb{Z})$ and each of them is simple except when $\alpha=0$ which is a double root. If we pick $\lambda$ a Liouville number, as done by Leont'ev, these roots tend to pair up, at least for infinitely many values of $p$ and $q$. In fact, with a convenient choice of $\lambda$, one can find subsequences $\mathrm{p}_{k}, \mathrm{q}_{k}$ of the integers such that

$$
\left|\mathrm{p}_{\mathrm{k}}-\mathrm{q}_{\mathrm{k}} \lambda\right| \leq\left(1+\left|\mathrm{p}_{\mathrm{k}}\right|\right)^{-\mathrm{k}} \text { as } \mathrm{k} \rightarrow \infty
$$

and these values of the frequencies cause trouble for the unrestricted convergence of the series (5) (compare with Theorems 4 and 5 below). Other important work on difference-differential equations appeared in $[12,17,68,69,73]$. An account of these and other references can be found in [58, Section 1] and in the treatise [1, especially chapters 4 and 6$]$.

Meanwhile, in 1935, Delsarte, inspired by the work of Fredholm, Pincherle and Volterra (see for instance [70]), had considered more general convolutors $\mu$ and coined the term mean-periodic function. This terminology arose from the fact that if $\mathrm{f}$ satisfies

$$
\mu * \check{f}(x)=\int_{x-\pi}^{x+\pi} f(t) d t \equiv 0,
$$

then $f$ is periodic and its average (mean) over a whole period is zero [15]. 
Finally, in 1947, L. Schwartz succeeded in proving the following version of Theorem 1 for functions of one variable $(f \in E(\mathbb{R}))$.

THEOREM 2 (see [64]): Let $f$ be mean-periodic with respect to $\mu$ and let $V=V(\hat{\mu})=\{\alpha \in C: \hat{\mu}(\alpha)=0\}$ be the zero set of $\hat{\mu}$. Then $f$ has a unique formal series expansion (5) and there are disjoint finite subsets $\mathrm{v}_{\mathrm{k}}$ (groupings) such that $\mathrm{V}=\bigcup_{1}^{\infty} \mathrm{V}_{\mathrm{k}}$ and

$$
\left.\sum_{k=1}^{\infty} \sum_{\alpha \in V_{k}} c_{\alpha}(x) e^{-i \alpha x}\right)
$$

is actually convergent in $E$ to $f$, after a certain abelian summation procedure is performed. (For some details about this summation procedure, see next section. It can be interpreted as $f(x)=\lim \sum_{\varepsilon \rightarrow 0}^{\infty}\left(\sum_{\alpha=1} c_{\alpha, \varepsilon}(x) e^{-i \alpha x}\right)$, where both the series and the limit are meaningful in the topology of $E$.

From this theorem it follows that if $f$ satisfies several convolution equations, the only non-zero coefflclents $c_{\alpha}(x)$ in (5) are those indexed by frequencies $a$ which are common roots of the Fourier transforms of those convolutions. In particular we have

COROLLARY: (Spectral Analysis Theorem) If $f$ satisfies the convolution equations

$$
\mu_{1} * \check{\mathrm{f}}=\mu_{2} \star \check{\mathrm{f}}=\cdots=\mu_{r} * \check{\mathrm{f}}=0 \text {, }
$$

and

$$
\left\{\alpha \in \mathbb{C}: \hat{\mu}_{1}(\alpha)=\cdots=\hat{\mu}_{r}(\alpha)=0\right\}=\emptyset \text {, }
$$

then $\mathrm{f} \equiv 0$.

This is a generalization of the fact that a non-constant function cannot have two incommensurable periods. 
In the fifties, J-P. Kahane (in his thesis [40]) and A. F. Leont'ev extended Schwartz' results to analytic mean-periodic functions. An account of Leont'ev's work in this area accompanied by a detailed bibliography appears in [48]. In [21], Ehrenpreis showed that for a wide class of distributions $\mu$ the abelian summation process is not necessary. More precisely, we call a distribution $\mu \in E^{\prime}$ slowly decreasing if there are constants $\mathrm{A}, \mathrm{B}, \varepsilon>0$ such that for any $\mathrm{x} \in \mathbb{R}$

$$
\max \{|\hat{\mu}(y)|: \quad y \in \mathbb{R},|x-y| \leq A \log (2+|x|)\} \geq \varepsilon(1+|x|)^{-B} \text {. }
$$

It turns out that every $\mu$ for which $\hat{\mu}$ is a non-zero exponential-polynomial is slowly decreasing. On the other hand, if $\mu \in C_{0}^{\infty}$ then it is not slowly decreasing (see $[21,5]$ ).

THEOREM 3 [21]: If $\mu$ is slowly decreasing, there exist finite groupings $v_{k}$ of $V(\hat{\mu})$ so that for any $f \in E$ satisfying $\mu * \check{\mathrm{f}}=0$ the corresponding series

$$
f(x)=\sum_{k}\left(\sum_{\alpha \in V_{k}} c_{\alpha}(x) e^{-i \alpha x}\right)
$$

converges to $f$ in $E$.

So far, we have considered only mean-periodic functions in $E$, but it is, of course, possible to replace $E$ by other spaces. Schwartz actually defined continuous mean-periodic functions; i.e., $f \in C\left(\mathbb{R}^{n}\right)$ is mean-periodic if there is a non-constant Borel measure $\mu$ with compact support such that $\mu * f \equiv 0$. However, such $f$ can be approximated very well by functions $E\left(\mathbb{R}^{n}\right) \ni f_{\varepsilon}=\chi_{\varepsilon} * f \rightarrow f$, where $\chi_{\varepsilon}(x)=\varepsilon^{-n} \chi\left(\frac{x}{\varepsilon}\right)$ is, as usual, a $c^{\infty}$ sequence tending to $\delta_{0}$, and these functions also satisfy the same equation,

$$
\mu * \mathrm{f}_{\varepsilon}=\mu * \mathrm{X}_{\varepsilon} * \mathrm{f}=(\mu * \mathrm{f}) * \mathrm{X}_{\varepsilon}=0,
$$

by the commutativity and associativity of the convolution product. Thus, the 
theory of continuous mean-periodic functions can be reduced to the case of smooth functions and is not studied separately. Similarly, if $E$ is replaced by $\theta^{\prime}$, the space of all distributions, then one obtains mean-periodic distributions. By considering instead of $E$, the space $A\left(c^{n}\right)=\left\{f\right.$ entire holomorphic in $\left.c^{n_{n}}\right\}$ and convolutors $\mu \in A^{\prime}\left(c^{n}\right)$ (analytic functionals, basically Radon measures with compact support in $\mathbf{c}^{\mathrm{n}}$ ) one has the analytic mean-periodic functions. Generally, the reasoning involved carries over from $E$ to these other cases and only occasionally will we point out some differences as remarks. Accordingly, functions $f$ will always be smooth and distributions $\mu$ always have compact support unless otherwise stated.

REMARK 1: There is a technical condition similar to (9), defining slowly decreasing analytic functionals. It is easy to see that every $\mu \neq 0$ is automatically slowly decreasing in this sense, hence the conclusion of Theorem 3 holds with no restrictions in the case of analytic mean-periodic functions. (The convergence of (10) being this time in $A(\boldsymbol{C})$, see $[7,23]$.

Neither of Theorems 2 and 3 provides information analogous to parts (ii) and (iii) of Theorem 1. Nor do they address the question of characterizing these slowly decreasing $\mu$ for which the series (5) converges in $E$ without any group-

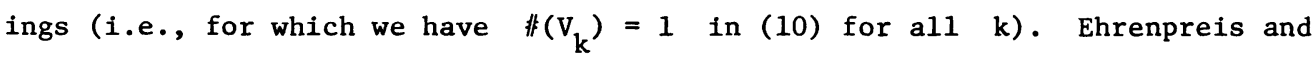
Malliavin gave partial answers to both questions in [24]. We shall give the complete answer in the next section.

For the more subtle properties of mean-periodic functions, such as their relation to almost-periodicity, quasianalyticity, and related questions in the theory of Dirichlet series, see $[1,10,20,23,26,41,42,51,52,53,55,65,66,67]$.

3. We continue to deal with the case of one variable. Under the assumption that $\mu$ is slowly decreasing, we shall state a theorem that extends Theorem 1 and give a sketch of its proof. Under the same assumption on $\mu$ we characterize convergence 
without groupings. To end the section we indicate how to prove Theorem 2 .

Given $n$ numbers $a_{1}, \ldots, a_{n}$, associate to them a norm in $c^{n}$ as follows. Let $d$ denote $d=\max \left\{1,\left|a_{k}-a_{j}\right|, \quad 1 \leq k, j \leq n\right\}$. We assume first that all the $a_{j}$ are distinct. Then if $b \in \mathbb{C}^{\mathrm{n}}$ we define

$$
\|b\|=\left(\sum_{j=1}^{n}\left|c_{j} d^{-j}\right|^{2}\right)^{\frac{1}{2}},
$$

where the vector $c=\left(c_{1}, \ldots, c_{n}\right)$ is related to $b$ by $c=D b, D$ the nonsingular matrix

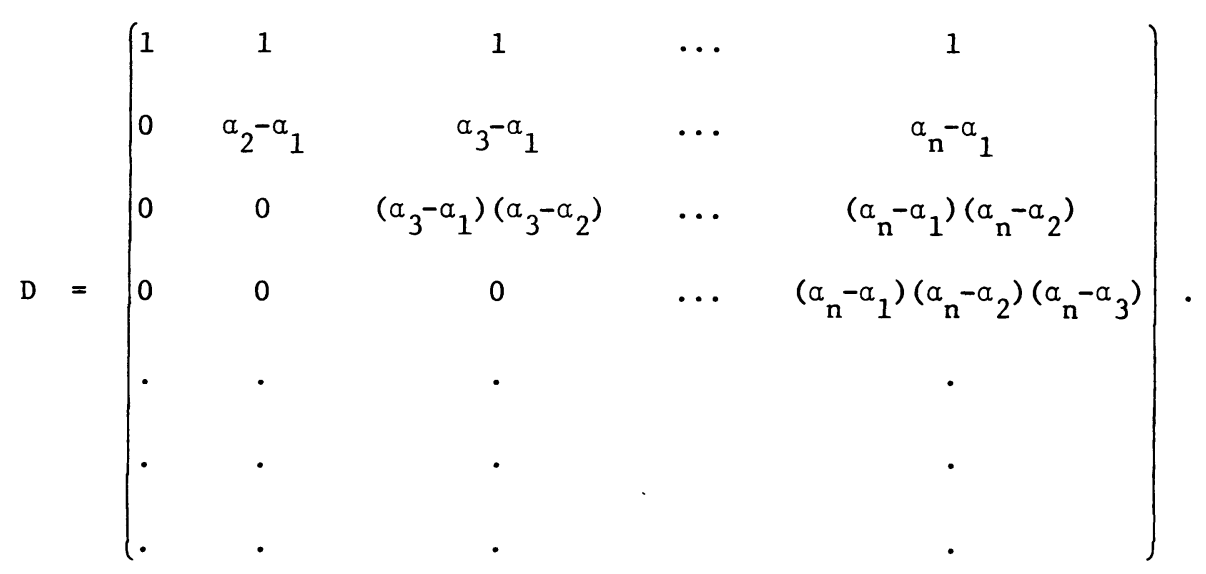

The transpose of this matrix appears as the matrix of the linear map $\left(b_{1}, \ldots, b_{n}\right) \rightarrow$ $\left(Q\left(a_{1}\right), \ldots, Q\left(a_{n}\right)\right)$ where $Q$ is the Newton interpolation polynomial

$$
Q(z)=\sum_{j=1}^{n} b_{j} \prod_{1 \leq k<j}\left(z-a_{k}\right)=b_{1}+b_{2}\left(z-a_{1}\right)+\cdots .
$$

It is not hard to see what to do when some of the $a_{j}$ coincide. For example, consider the sequence $a_{1}, a_{2}, a_{2}, a_{2}, a_{3}$, and the map $\left(b_{1}, \ldots, b_{5}\right) \rightarrow\left(Q\left(a_{1}\right)\right.$, $\left.Q\left(\alpha_{2}\right), Q^{\prime}\left(\alpha_{2}\right), \frac{Q^{\prime \prime}\left(\alpha_{2}\right)}{2 !}, Q\left(\alpha_{3}\right)\right)$; the corresponding $5 \times 5$ matrix $D$ is given by 


$$
D=\left(\begin{array}{ccccc}
1 & 1 & 0 & 0 & 1 \\
0 & a_{2}-a_{1} & 1 & 0 & a_{3}-a_{1} \\
0 & 0 & a_{2}-a_{1} & 1 & \left(a_{3}-a_{1}\right)\left(a_{3}-a_{2}\right) \\
0 & 0 & 0 & a_{2}-a_{1} & \left(a_{3}-a_{1}\right)\left(a_{3}-a_{1}\right)^{2} \\
0 & 0 & 0 & 0 & \left(a_{3}-a_{1}\right)\left(a_{3}-a_{2}\right)^{3}
\end{array}\right) .
$$

As in Theorem 3, to the grouping $v_{k}$ of roots $a_{1}, \ldots, a_{r}$ in $v(\hat{\mu}) \quad\left(r=r_{k}\right)$ we will associate the partial sum of (5)

$$
\sum_{\alpha \in V_{k}} c_{\alpha}(x) e^{i \alpha x}=\sum_{j=1}^{r}\left(\sum_{\ell=0}^{m_{j}-1} b_{j, \ell} \frac{(-i x)^{\ell}}{\ell !}\right) e^{-i \alpha{ }_{j} x},
$$

where $m_{j}$ indicates the multiplicity of the root $a_{j}$. Set $n=n_{r}=m_{1}+\cdots+$ $\mathrm{m}_{\mathrm{r}}, \quad \mathrm{b}=\left(\mathrm{b}_{1,0}, \ldots, \mathrm{b}_{1, \mathrm{~m}_{1}-1}, \mathrm{~b}_{2,0}, \ldots, \mathrm{b}_{\mathrm{r}, \mathrm{m}_{\mathrm{r}}-1}\right) \in \mathrm{c}^{\mathrm{n}}$, and let $\|\mathrm{b}\|_{(\mathrm{k})}$ denote the norm (11) of this vector $b$ computed using the sequence $a_{1}, \ldots, a_{1}, a_{2}, \ldots, a_{r}$ where each root appears repeated according to its multiplicity and $d=d_{k}=$ $\max \left\{1,\left|\alpha_{j}-a_{\ell}\right|, \quad 1 \leq j, \quad \ell \leq r\right\}$.

We can now state the main result of this section. Set $p(z)=$ $|\operatorname{Im} z|+\log (1+|z|)$ to simplify the writing.

THEOREM 4 [7]: Let $\mu$ be slowly decreasing. Then the zero set $V$ of $\hat{\mu}$ can be divided into disjoint finite groupings $V_{k}, \quad V=\{\alpha \in \mathbf{C}: \hat{\mu}(\alpha)=0\}=\bigcup_{k=1}^{\infty} V_{k}$, in such a way that any mean-periodic function $f$ with respect to $\mu$ has a Fourier expansion (10) convergent in $E$. Furthermore, the coefficients of the expansion, as defined by (14), satisfy the estimates

$$
\|b\|_{(k)}=O\left(\exp \left(-N p\left(\alpha_{k}\right)\right)\right) \text { as } k \rightarrow \infty \text {, }
$$

for any $N>0$. Here $a_{k}$ denotes any point in $v_{k}$. Conversely, any series (10) whose coefficients satisfy (15) defines a function $f$ in $E$ such that $\mu * \check{f}=0$. 
SKETCH OF THE PROOF: The idea goes back to work of Ehrenpreis on partial differential equations which we shall discuss in Section 6 [4, 23, 59]. As everywhere throughout the theory, the fundamental tool is the Fourier transform,

$$
\hat{\mu}(z)=\left\langle\mu_{x}, e^{-i x z}\right\rangle \text {, }
$$

of distributions of compact support. We also need to use the Paley-WienerSchwartz theorem which states that the map $F: \mu \rightarrow \hat{\mu}$ is a linear isomorphism between $E^{\prime}$ and the space of entire functions $\hat{L}^{\prime}$

$$
\hat{E}^{\prime}:=\left\{\varphi: \exists A \geq 0 \text { such that }|\varphi(z)| \leq A(1+|z|)^{A} \exp (A|\operatorname{Im} z|)\right\} \text {, }
$$

where $|z|=(z \cdot \bar{z})^{\frac{1}{2}}=\left(\left|z_{1}\right|+\cdots+\left|z_{n}\right|^{2}\right)^{\frac{1}{2}}$ and $\operatorname{Im} z=\left(\operatorname{Im} z_{1}, \ldots, \operatorname{Im} z_{n}\right)$. The convolution product corresponds to the usual multiplication of functions under this isomorphism. The space $\hat{E}^{\prime}$ is the inductive limit of the Banach spaces $\hat{E}_{N}^{\prime}$,

$$
\hat{E}_{N}^{\prime}:=\left\{\varphi \text { entire: } \sup _{z}|\varphi(z)|(1+|z|)^{-N} \exp (-N|\operatorname{Im} z|)<\infty\right\}
$$

Ehrenpreis proved that the Fourier transform map $F$ is also a topological isomorphism [23]. It is this last result that permits the reduction of questions about mean-periodic functions to problems in function theory.

To outline the proof, let $M$ be the closed subspace of all mean-periodic functions with respect to $\mu$,

$$
M=\{f \in E: \mu \star \check{f}=0\} .
$$

The ideal generated by $\mu, J=\left\{\mu * \nu: \nu \in E^{\prime}\right\}$ is closed in $E^{\prime}$ because $\mu$ is slowly decreasing [21]. It annihilates $M$ (recall $\langle\mu * \nu, f\rangle=\nu * \mu * \check{f}(0))$, and one can see that $M$ is precisely the dual of the space $E^{\prime} / J$ (see [38]). Using the Fourier transform isomorphism, one sees that this last space is isomorphic to 
the quotient space $\hat{E}^{\prime} / \hat{\jmath}$, where $\hat{g}$ is the ideal generaged by $\hat{\mu}$ in $\hat{E}^{\prime}$. On the other hand, if $\varphi$ is any function in $\hat{E}^{\prime}$ and $[\varphi]$ its class modulo $\hat{J}$, then [ $\varphi$ ] is completely determined by the restriction of $\varphi$ to $V$. (By restriction we mean the double-indexed sequence

$$
\frac{\varphi^{(\ell)}}{\ell !}(\alpha), \quad 0 \leq \ell<\mathrm{m}
$$

where $a \in \mathrm{V}$ has multiplicity $\mathrm{m}$. Such a double-indexed sequence $\left\{a_{\alpha, \ell}\right\}$ constitutes an analytic function on v.) The proof is completed by characterizing those analytic functions on $V$ which are restrictions of $\varphi \in \hat{E}^{\prime}$. (Here is where the interpolation theory mentioned in the introduction enters.) Once this is accomphished, an element $f \in M$ defines a continuous linear functional $F$ on this space, and so can be identified with a double-indexed sequence $\left\{b_{a, \ell}\right\}$. This defines the series (10) we are looking for, in fact,

$$
f(x)=<\delta_{x}, f>=F_{z}\left(e^{-i z x}\right)=\sum_{\alpha \in V} \sum_{\ell=0}^{m-1} \frac{(-i x)^{\ell}}{\ell !} b_{\alpha, \ell} e^{-i \alpha x},
$$

since the restriction to $v$ of $e^{-i z x}=F\left(\delta_{x}\right)$ is given by the sequence $\frac{(-i x)^{\ell}}{\ell !} e^{-i a_{x}}$. The fact that the series (5) obtained from this converges absolutely, after groupings, in $E$ and the required estimates (15) are consequences of the above mentioned characterization of $\hat{E}^{\prime} / \hat{J}$ as a certain space of analytic functions on $V$ (see [7] for the details).

REMARK 2: In the case of analytic mean-periodic functions there is no restriction on $\mu$ (see Remark 1) and Theorem 4 holds if we take $p(z)=|z|$ in (15). Note that in the last theorem we gave no indication of the size of the groupings $v_{k}$, i.e. of the numbers $r_{k}$ and $n_{k}$ appearing in the definition of the norm $\|\cdot\|_{(k)}$. Actually such an estimate exists (see [7]) but $r_{k}$ and $n_{k}$ are unbounded in general. In the case of difference-differential operators, it can be 
seen that $d_{k}$ can be taken $\leq 1$ and $n_{k}$ is always bounded, in fact, if $\hat{\mu}$ is given by (6) and $\nu_{j}$ indicates the degree of the polynomial $a_{j}$, one gets the estimate

$$
\mathrm{n}_{\mathrm{k}} \leq \nu+\frac{2(1+\nu)}{\log v}(1+\Omega)
$$

where $v=\sum_{j}\left(1+v_{j}\right), \Omega=\max _{j}\left|\omega_{j}\right|$ (see [7]). In the case of the example (7) the zeroes $a_{j}$ are all simple (with only the exception $\alpha=0$ ), $n_{k}=r_{k} \leq 2$ but in general $r_{k} \neq 1$ as can be seen from Theorem 5 below.

In the next section we give an application of the estimates (15). Here we will compare them with classical. ones for Fourier series given by Theorem 1 (ii). If all the $r_{k}=1$ (see Theorem 5), then the norms $\|\cdot\|_{(k)}$ are easy to compute. In fact, in this case the multiplicity $m_{k}$ of $a_{k}$ coincides with $n_{k}$ and

$$
f(x)=\sum_{k}\left(\sum_{j=0}^{m_{k}-1} \frac{(-i x)^{\ell}}{\ell !} b_{k, \ell}\right) e^{-i \alpha_{k} x}
$$

is absolutely convergent over compact sets, etc. and for any $\mathrm{N}>0$

$$
\left|b_{k, \ell}\right|=O\left(\exp \left(-\mathrm{Np}\left(a_{k}\right)\right)\right) \text { as } k \rightarrow \infty \text {. }
$$

In the case of the Fourier series the roots are $\alpha_{k}=k \in \mathbf{z}, \quad r_{k}=n_{k}=1$ and (16) is exactly (ii) of Theorem 1 since $p(k)=\log (1+|k|)$. The estimate (16) is the same obtained by Ehrenpreis-Malliavin [24], where only the case $r_{k} \equiv 1$ was discussed.

It is easy to derive interesting and simple estimates from (16) even when $r_{k}>1$. For example, if we assume $d_{k}=1$ and all the multiplicities $m_{1}=\ldots=m_{r}=1 \quad\left(r=r_{k}\right)$, we can write the grouping $v_{k}$ as $a_{1}^{(k)}, \ldots, a_{r}^{(k)}$, 


$$
f(x)=\sum_{k}\left(\sum_{j=1}^{r} b_{j}^{(k)} \exp \left(-i \alpha_{j}^{(k)} x\right)\right),
$$

and (16) implies that for $j=1, \ldots, r$

$$
\left|b_{j}^{(k)}\right| \prod_{\ell \neq j}\left|\alpha_{\ell}^{(k)}-a_{j}^{(k)}\right|=0\left(\exp \left(-N p\left(\alpha_{j}^{(k)}\right)\right)\right)
$$

for every $N>0$ (see [7]).

THEOREM 5 [7]: Let $\mu$ be slowly decreasing. A necessary and sufficient condition that every grouping $v_{k}$ can be taken to contain a single point of $v$ (i.e. $\mathbf{r}_{\mathbf{k}} \equiv 1$ ) is that for some $\varepsilon, \mathrm{C}>0$

$$
\left|\hat{\mu}^{(m)}(\alpha)\right| \geq \varepsilon \frac{\exp (-c|\operatorname{Im} \alpha|)}{(1+|\alpha|)^{c}}
$$

where $\alpha$ runs after all the points of $V$ and $m$ is the multiplicity of $a$ as a root of the equation $\hat{\mu}(\alpha)=0$. (As usual $\hat{\mu}^{(m)}(\alpha)$ denotes $\left.\frac{d^{m}}{d z^{m}} \hat{\mu}(z)\right|_{z=\alpha}$.)

While Theorem 5 is not stated explicitly in [7] it is an immediate consequence of Example 17 and Remark 4 there.

Ehrenpreis-Malliavin [24] give a condition immediately seen to be equivalent to (17) in the only case they considered, $m_{k} \equiv 1$.

REMARK 3: In the analytic case (17) is replaced by

$$
\left|\frac{\hat{\mu}^{(m)}(\alpha)}{m !}\right| \geq \varepsilon \frac{\exp (-c|\alpha|)}{(1+|\alpha|)^{m}} .
$$

Within the class of difference-differential operators, it is easy to see that if (6) has all frequencies $\omega_{j}$ rational (or mutually commensurable) then there is no need for groupings (i.e. $r_{k} \equiv 1$ ). On the other hand, the example (7) shows that this is not always the case (the roots get too close together because of the transcendental nature of the relation between the frequencies.) Since the 
multiplicities $m_{k}$ are bounded in the case of exponential polynomials, (17) shows that a necessary condition for $r_{k} \equiv 1$ is that for some $\delta, A>0$

$$
\left|a_{k}-a_{j}\right| \geq \delta \frac{\exp \left(-A\left|\operatorname{Im} a_{k}\right|\right)}{\left(1+\left|a_{k}\right|\right)^{A}}, \quad(k \neq j) .
$$

It follows easily from the estimates in [5, 31] or even from [17] that (19) is also sufficient for $r_{k} \equiv 1$. Furthermore, from the work of Polya et a1 [17, 46], it follows that (19) is equivalent to the existence of $\eta$, B > 0 such that

$$
\left|a_{k}-a_{j}\right| \geq \eta\left(1+\left|\alpha_{k}\right|\right)^{-B}, \quad(k \neq j)
$$

Ehrenpreis has conjectured that if all the $\omega_{j}$ are algebraic then (20) holds [23]. Particular cases of this conjecture have been proved [22, 57]. This discussion also shows that Theorem 5 includes the results from [72]. (Certainly, the consequences of Theorem 5 for difference-differential equations when (19) holds have been part of the folklore of the subject and motivated Ehrenpreis' conjecture.)

It has recently been shown by $W$. A. Squires (private communication) that the condition, $\mu$ slowly decreasing, is not necessary for the validity of Theorem 4 . In general, we can remove it at the expense of bringing into the picture a further summation procedure. Our method is similar to that used by Schwartz in [64, 66] to prove Theorem 2; but, since we avoid reliance on the uniqueness of the coefficients of the Fourier expansion, we can handle the case of more than one variable (where this uniqueness does not hold). Roughly the idea consists in noting that: (i) in dealing with the analytic case there is no restriction at all on $\mu$ (other than $\mu \neq 0$ ) for convergence with groupings, and (ii) given $\mu \neq 0$ and $\varepsilon>0$ there is a constant $c>0$ such that for $x \in \mathbb{R}$

$$
\max \{|\hat{\mu}(y)|: \quad y \in \mathbb{R} \text { and }|x-y| \leq(1+\varepsilon|x|)\} \geq C e^{-\varepsilon|x|}
$$


(compare with (9)). Thus although not every $\mu \in E^{\prime}$ is slowly decreasing, they fail by very little. This fact has been used in other contexts [33, 37]; here the idea is to use (ii) to reduce the situation to one similar to (i).

Let $f \in E$ satisfy the equation $\mu * \check{\mathrm{f}}=0$, and choose $\mathrm{L}_{0}>0$ so that supp $\mu \subseteq\left(-\mathrm{L}_{0}, \mathrm{~L}_{0}\right)$. In what follows $\mathrm{R}>0$ will be a sufficiently large constant (eventually we let $R \rightarrow+\infty$ ). Call the function that coincides with $f$ in $(-R, R)$ and is identically zero outside this interval $f_{R}$; then

$$
\mu * \check{f}_{R}(x)=0 \text { when } x \in\left(-R+L_{0}, R-L_{0}\right) \text {. }
$$

Since $f_{R}$ is bounded and of compact support, its Cauchy transform is defined as

$$
F_{R}(\zeta)=\frac{1}{2 \pi i} \int_{-R}^{R} \frac{f(t)}{\zeta-t} d t
$$

for $\zeta \in \mathbf{C}, \quad \operatorname{Im} \zeta \neq 0 \quad([13,14,54])$. We get two analytic functions $\mathrm{F}_{\mathrm{R}^{+}}^{+} \mathrm{F}_{\mathrm{R}}^{-}$ in $\operatorname{Im} \zeta>0$ and $\operatorname{Im} \zeta<0$ respectively. The function $f_{R}$ can be recovered from the pair $\mathrm{F}_{\mathrm{R}^{\prime}}^{+}, \mathrm{F}_{\mathrm{R}}^{-}$by

$$
\lim _{y \rightarrow 0+}\left[F_{R}^{+}(x+i y)-F_{R}^{-}(x-i y)\right]=f(x), \quad|x|<R .
$$

The limit makes sense in $E(|x|<R)$, i.e., is uniform over compact subsets of $(-R, R)$ and the same is true for derivatives.

The distribution $\mu$ acts as a convolutor also on functions holomorphic in the upper half-plane by means of the formula

$$
\mu * \varphi(z)=\int \varphi(z-t) \mu(t) d t=<\mu_{t}, \varphi(z-t)>,
$$

where the "integration" takes place over the real axis, the function $\mu * \varphi$ is also holomorphic for $\operatorname{Im} z>0$. Similarly for $\operatorname{Im} z<0$ or even if $\varphi$ is 
defined only on a strip parallel to the real axis. If $\varphi$ is defined on a vertical strip or half-strip, then $\mu * \varphi$ still makes sense as long as the width of this strip is bigger than $\mathrm{L}_{0}$ and $z$ is restricted conveniently. Since $\mu * \check{\mathrm{f}}(\mathrm{x})=\check{\mu} * \mathrm{f}(-\mathrm{x})$, it is easy to see that (22) implies

$$
\lim _{\mathrm{y} \rightarrow 0+}\left[\left(\check{\mu} * \mathrm{~F}_{\mathrm{R}}^{+}\right)(\mathrm{x}+i \mathrm{y})-\left(\check{\mu} * \mathrm{~F}_{\mathrm{R}}^{-}\right)(\mathrm{x}-i \mathrm{y})\right]=0,|\mathrm{x}|<\mathrm{R}-\mathrm{L}_{0},
$$

and the limit is uniform on compact subsets of the above interval. Hence the functions $\check{\mu} * \mathrm{~F}_{\mathrm{R}}^{+}$and $\check{\mu} * \mathrm{~F}_{\mathrm{R}}^{-}$are holomorphic continuation of each other across the segment $\left(-R+L_{0}, R-L_{0}\right)$ of the real axis, and they define a holomorphic function $G_{R}$ in the vertical strip $\left\{z \in C:|R e z|<R-L_{0}\right\}$. Because of the estimate (21) one can find $H_{R}$ holomorphic in $|R e z|<R$ such that

$$
\check{\mu} * H_{R}=G_{R} \text {, when }|\operatorname{Re} z|<R-L_{0} \text {. }
$$

Hence

$$
\check{\mu} *\left(\mathrm{~F}_{\mathrm{K}}^{ \pm}-\mathrm{H}_{\mathrm{R}}\right)=0,
$$

for $|\operatorname{Re} z|<\mathrm{R}-\mathrm{L}_{0}$ and $\operatorname{Im} z>0$ or $\operatorname{Im} z<0$ respectively. The same kind of argument used in Theorem 4 shows that for $R$ big enough there are groupings $V_{k}$ independent of $R$ and $L_{1}>0$, also independent of $R$, such that

$$
F_{R}^{ \pm}(z)-H_{R}(z)=\sum_{k}\left(\sum_{\alpha \in V_{k}} c_{\alpha, R}^{ \pm}(z) e^{-i \alpha z}\right)
$$

with uniform and absolute convergence in compact subsets of $|\operatorname{Re} z|<R-L_{1}$, $\operatorname{Im} z>0$ or $\operatorname{Im} z<0$ respectively, where the notation is self-explanatory. Since we also have

$$
\lim _{y \rightarrow 0+}\left[\left(F_{R}^{+}-H_{R}\right)(x+i y)-\left(F_{R}^{-}-H_{R}\right)(x-i y)\right]=f(x)
$$


in $E(|x|<R)$ from (23), we can apply a diagonal process and obtain polynomials $c_{\alpha, \varepsilon}$ such that

$$
f(x)=\lim _{\varepsilon \rightarrow 0+} \sum_{k}\left(\sum_{\alpha \in V_{k}} c_{\alpha, \varepsilon}(x) e^{-i \alpha x}\right)
$$

where the convergence of the series and of the limit is in $E(\mathbb{R})$. This gives a proof of Theorem 2 which avoids involving the uniqueness of the coefficients or the Spectral Analysis Theorem. Using these results, one sees easily that the $c_{\alpha, \varepsilon} \rightarrow c_{\alpha}$ when $\varepsilon \rightarrow 0$

3. We give here a simple application of the estimates (15) from Theorem 4. This example is also borrowed from [7] but we provide more details here.

We wish to study the problem, raised in [21], of solving the over-determined system of convolution equations: $\mu, \nu \in E^{\prime}$

$$
\left\{\begin{array}{l}
\mu * \mathrm{f}=\mathrm{g} \\
\nu * \mathrm{f}=\mathrm{h}
\end{array}\right.
$$

where $g, h \in E$ and the unknown function $f$ is also sought in $E$. Clearly, one has to assume the compatibility condition

$$
\mu * h=\nu * g
$$

and we do so henceforth. To simplify the arguments we assume there is no "resonance" in this system, that is,

$$
\{\alpha \in C: \hat{\mu}(\alpha)=\hat{\nu}(\alpha)=0\}=\emptyset \text {. }
$$

In order to apply Theorem 4 we assume that one of the distributions, say $\mu$, is slowly decreasing. (A11 these assumptions were already made in [21].) Under the above assumptions we wish to find necessary and sufficient conditions on the pair 
$\mathrm{g}, \mathrm{h}$ in order that the system (25) have a solution in $E$. Before settling this, let us show how functional analysis provides the answer to the related question, for which $\mu, \nu$ is it possible to solve (25) for all $g, h$ which satisfy (26)? First of all, note that the lack of resonance together with the Spectral Analysis Theorem show that (a) if a solution $f$ to (25) exists at all, it is unique, and (b) the algebraic ideal $((\mu, \nu))$ generated in $E^{\prime}$ by $\mu$ and $\nu$ is dense in $E^{\prime}$. (If $f \in E$ annihilates $((\mu, \nu)$ ) then it necessarily satisfies $\mu * \mathrm{f}=\nu * \underset{\mathrm{f}}{=0}$ and hence $\mathrm{f}=0$ by (a); since $E$ is reflexive, (b) follows.) If $((\mu, \nu))$ were closed then by (b) there would be distributions $\mu_{0}, \nu_{0} \in E^{\prime}$ such that

$$
\mu * \mu_{0}+\nu * \nu_{0}=\delta
$$

Conversely, (28) implies that $((\mu, \nu))=E^{\prime}$ and hence it is closed. It is easy to check that $f=\mu_{0} * g+\nu_{0} * h$ solves (25), hence (28) is sufficient for the solvability of (25) for every pair that satisfies (26). We now show that (28) is also a necessary condition. The set $K$ of pairs $(g, h)$ satisfying $(26)$ is a closed subspace of the Fréchet space $E \oplus E$. Let $M$ denote the 1-1 map

$$
M: f \longrightarrow(\mu * f, \nu * f)
$$

If image $(M)=K$ then, by the open mapping theorem [38], $M^{\prime}: E^{\prime} \uplus E^{\prime} \rightarrow E^{\prime}$ is onto, where $M^{\prime}$ is the transpose map to $M$. Now,

$$
\begin{aligned}
<\mathrm{M}^{\prime}(\varphi, \psi), \mathrm{f}_{>} & =\left\langle(\varphi, \psi), \mathrm{Mf}>=\left\langle\varphi, \mu * \mathrm{f}_{>}+\left\langle\psi, \nu * \mathrm{f}_{>}\right.\right.\right. \\
& =\varphi * \check{\mu} * \check{\mathrm{f}}(0)+\psi * \check{\nu} * \check{\mathrm{f}}(0) \\
& =<\psi * \check{\mu}+\psi * \check{\nu}, \mathrm{f}_{>},
\end{aligned}
$$

hence $M^{\prime}(\varphi, \psi)=\varphi * \check{L}+\psi * \check{v}$. Since $M^{\prime}$ is onto (28) follows.

If follows from [34, 40] that (28) is equivalent to the existence of constants 
$\varepsilon, \quad C>0$ such that

$$
|\hat{\mu}(z)|+|\hat{\nu}(z)| \geq \varepsilon \frac{\exp (-C|\operatorname{Im} z|)}{(1+|z|)^{C}} \text {, for all } z \text {. }
$$

Going back to our original problem, it is clearly interesting only when (29) fails, e.g. $\hat{\mu}(z)=\sin \pi z$ and $\hat{v}(z)=\frac{1}{z} \sin \frac{\pi}{\lambda} z$, where $\lambda$ is chosen as in (8) above. Note that we can reduce the pair of equations (25)-(26) to the equivalent

$$
\left\{\begin{array}{l}
\mu * F=0 \\
\nu * F=H
\end{array}\right.
$$

with the compatibility condition

$$
\mu * \mathrm{H}=0 \text {. }
$$

This is possible since $\mu$ being slowly decreasing implies $\mu *: E \rightarrow E$ is onto [21], hence we can find $G \in E$ such that $\mu * G=g$ and take $F=f-G, H=h-\nu * G$ in $(30)-(31)$. It follows that $\check{F}$ and $\check{\mathrm{H}}$ are mean-periodic with respect to $\mu$, so we can apply Theorem 4, obtaining

$$
F(x)=\sum_{k}\left(\sum_{\alpha \in V_{k}} \varphi_{\alpha}(-x) e^{i \alpha x}\right)
$$

and

$$
H(x)=\sum_{k}\left(\sum_{\alpha \in V_{k}} \eta_{\alpha}(-x) e^{i \alpha x}\right),
$$

both convergent in $E$. Applying the second equation from (3), we get

$$
\check{v} *\left(\varphi_{\alpha} e^{-i \alpha x}\right)=\eta_{\alpha} e^{-i \alpha x},
$$

which leads to a triangular system of equations in the coefficients of the 
polynomials $\varphi_{\alpha}$. This linear system is solvable (uniquely) because all the terms in the diagonal equal $\hat{v}(\alpha)$, which doesn't vanish since $\hat{\mu}(\alpha)=0$ and we have assumed (27) holds. Applying the conditions (15) to the coefficients of F obtained this way, we obtain a set of necessary and sufficient conditions in the coefficients of $\mathrm{H}$ for the solvability of the system (30). These conditions become simpler when all the roots of $\hat{\mu}(\alpha)=0$ are simple, since then (32) defines the numerical coefficients $\varphi_{\alpha}$ by

$$
\varphi_{\alpha} \hat{\nu}(\alpha)=\eta_{a}
$$

If $a_{1}, \ldots, a_{r}$ form one of the groupings of roots of $\hat{\mu}(\alpha)=0$, and, for instance, $\max \left|\alpha_{j}-\alpha_{k}\right| \leq 1$ then, as explained before Theorem 5, a necessary condition on the $\eta_{\alpha}$ is given by

$$
\left|\eta_{a_{j}}\right| \prod_{k \neq j}\left|a_{k}-a_{j}\right|=\left|\hat{v}\left(a_{j}\right)\right| O\left(\exp \left(-\operatorname{Np}\left(a_{j}\right)\right)\right)
$$

for all $N>0 \quad(j=1, \ldots, r)$. For example, when $\hat{\mu}(z)=\sin \pi z$ and $\hat{v}(z)=$ $\frac{1}{z} \sin \frac{\pi}{\lambda} z$, we are really dealing with periodic functions $F$ and $H$ and one gets the condition on the Fourier coefficients $\eta_{k}$ of $\mathrm{H}$,

$$
\left|r_{k}\right|=O\left(|\hat{\nu}(k)|(1+|k|)^{-N}\right) \text { as } k \rightarrow \infty
$$

for every $N>0 \quad(\{\hat{\mu}=0\}=\mathbb{Z})$. This gives,

$$
\left|\eta_{k}\right|=O(1+|k|)^{-N} \underset{m \in \mathbb{Z}}{\operatorname{dist}(k, m \lambda))} \text { as } k \rightarrow \infty \text {, }
$$

which imposes a severe restriction on the Fourier coefficients of $H$ if $\lambda$ is chosen as in (8). 
5. We proceed now to discuss mean-periodic functions functions of several variables. It was the question of the existence of a Fourier representation for such functions which motivated both our work [8] and our reevaluation of the one variable situation, which in turn led to the Theorems 4 and 5 above. After the work of Schwartz [64], it wasn't very clear which shape, if any, Fourier representation would take in several variables. It was suggested in [64] that it might be easier to deal with the Spectral Analysis Theorem (and its companion, the Spectral Synthesis Theorem). Roughly speaking this corresponds to the uniqueness of the representation of a mean-periodic function in the case of one variable. However, it turned out that the Spectral Analysis Theorem fails when the number of variables $\mathrm{n} \geq 2$. In fact, in 1975, D. I. Gurevich constructed in [32] six distributions $\mu_{1}, \ldots, \mu_{6} \in E^{\prime}\left(\dot{\mathbb{R}}^{\mathrm{n}}\right) \quad(\mathrm{n} \geq 2)$ for which $\left\{z \in \mathbb{C}^{\mathrm{n}}: \hat{\mu}_{1}(\mathrm{z})=\cdots=\hat{\mu}_{6}(\mathrm{z})=0\right\}=\emptyset$ but $\left\{f \in E: \mu_{1} * \check{\mathrm{f}}=\cdots=\mu_{6} * \check{\mathrm{f}}=0\right\} \neq\{0\}$. (In the analytic case, one can get by with two equations.) On the positive side, Malgrange had shown in his thesis that the exponential-polynomial solutions to $\mu \star \check{\mathrm{f}}=0$ were, in fact, dense in the space of all solutions. This confirmed the possibility of a representation (see $[49,50]$ and $[19,37]$ for related results), but gave no hint as to what the representation looked like. In 1960, Ehrenpreis attacked this question in the case of partial differential operators with constant coefficients. To start with we shall see what can be done about the wave operator in two variables.

Since $E$ is an AU space, it follows that every smooth function has a Fourier representation of the following kind. Given $\varphi \in E\left(\mathbb{R}^{n}\right)$ there is a Radon Measure dv in $\mathbf{c}^{\mathbf{n}}$ such that

$$
\varphi(x)=\int_{\mathbb{C}^{n}} e^{-i x \cdot z} d \nu(z)
$$

and this integral converges in $E$ (see $[4,23]$ ). The point here is that we are allowing arbitrary complex frequencies to occur in (33). Going back to the wave 
equation in $\mathbb{R}^{2}$

$$
\left(\frac{\partial^{2}}{\partial x_{1}^{2}}-\frac{\partial^{2}}{\partial x_{2}^{2}}\right) f(x)=0
$$

D'Alembert showed that the general smooth solution of (34) is of the form

$$
f\left(x_{1}, x_{2}\right)=f_{1}\left(x_{1}-x_{2}\right)+f_{2}\left(x_{1}+x_{2}\right)
$$

with $f_{1}, f_{2}$ smooth functions of one variable (see [44]). Hence there are two measures $d \nu_{1}, d \nu_{2}$ in $\mathbb{C}$ such that

$$
f_{j}(t)=\int_{C} e^{-i t w} d \nu_{j}(w), \quad j=1,2,
$$

where $t$ denotes the variable in $\mathbb{R}$. Inserting (36) into (35) we get

$$
f(x)=\int_{C} e^{-i\left(x_{1}-x_{2}\right) w} d \nu_{1}(w)+\int_{C} e^{-i\left(x_{1}+x_{2}\right) w} d \nu_{2}(w)
$$

Let $z \in c^{2}$ represent the dual variable to $x \in \mathbb{R}^{2}$, then the above expression can be written as

$f(x)=\int_{\left\{z_{1}+z_{2}=0\right\}} e^{-i x \cdot z_{d}} v_{1}(z)+\int_{\left\{z_{1}-z_{2}=0\right\}} e^{-i x \cdot z_{d}} v_{2}(z)=\int_{\left\{z_{1}^{2}-z_{2}^{2}=0\right\}} e^{-i x \cdot z} d \nu(z)$,

where the last integral converges in $E$ and the Radon measure $d \nu$ is supported by the algebraic variety $v=\left\{z \in c^{2}: z_{1}^{2}-z_{2}^{2}=0\right\}$. This is precisely the algebraic variety $\mathrm{V}(\hat{\mu})=\left\{z \in \mathbf{c}^{2}: \hat{\mu}(z)=0\right\}$, where $\mu$ is the distribution arising from the convolution equation (34). (It is easy to see that the choice of measure dv is not unique [59].)

This representation of the solutions of the wave equation was generalized to 
the case of arbitrary linear differential operators with constant coefficients in [22]. As usual, if $P$ is a polynomial in $n$ variables with complex coefficients, and $D_{j}=\frac{1}{i} \frac{\partial}{\partial x_{j}}$, then $P(D)$ represents a partial differential operator.

THEOREM 6: Let $\mathrm{V}$ be the algebraic variety of the zeros of $P, V=\left\{z \in \mathbb{c}^{\mathrm{n}}\right.$ : $P(z)=0\}$. Then there are finitely many algebraic varieties $v_{j} \subset v, \underset{j=1}{U} v_{j}=v$, and differential operators $\partial_{j}$ with constant coefficients such that if $P(D) \check{f}=0$ then $f$ has a representation

$$
f(x)=\sum_{j=1}^{r} \int_{v_{j}} \partial_{j} e^{-i x \cdot z} d v_{j}(z)
$$

where the $d v_{j}$ are Radon measures in $\mathbb{C}^{\mathrm{n}}$ with support in $\mathrm{v}_{j}$; and all the integrals converge in $E$. Moreover, if all the irreducible factors of $P$ are distinct then one has the representation

$$
f(x)=\int_{V} e^{-i x \cdot z} d \nu(z),
$$

for some Radon measure $d \nu$.

The proof follows the same lines as Theorem 4 above except that the function theory needed is much harder. In [23, 59] this theorem is actually proved for systems. For instance, if $P_{1}(D) \check{f}=\cdots=P_{m}(D) \check{f}=0$ then (38) holds with these two differences (i) $V=\left\{z \in c^{n}: P_{1}(z)=\cdots=P_{m}(z)=0\right\}$ and (ii) the $\partial_{j}$ do no longer have constant coefficients. In particular, they show that the Spectral Analysis Theorem holds for partial differential operators, in contrast to the counterexample for general overdetermined systems found (later) by Gurevich. The point here is that the algebraic nature of the variety $V$ plays a very strong role. There is very little more we can offer by way of comment except to encourage the reader to look into the original sources. Recently some simplifications 
of parts of the proofs have appeared $[18,75,76]$.

In $[2,4]$ an extension of Theorem 6 was given for a subclass of those convolution operators $\mu$ which look like

$$
\mu * f(x)=\frac{\partial^{m}}{\partial x_{n}^{m}} f+\mu_{1}\left(x^{\prime}\right) * \frac{\partial^{m-1}}{\partial x_{n}^{m-1}} f+\cdots+\mu_{m}\left(x^{\prime}\right) * f,
$$

where $x^{\prime}=\left(x_{1}, \ldots, x_{n-1}\right)$ and $\mu_{1}, \ldots, \mu_{m} \in E^{\prime}\left(\mathbb{R}^{n-1}\right)$. The proof follows closely that of Ehrenpreis' Theorem 6 and avoids trouble by restricting consideration to a single equation. When [4] appeared, the remaining difficulties were twofold:

first, the concept of groupings had to be extended to several variables and, second, in the case of systems the Spectral Analysis Theorem had to be built into the system. We finally solved this problem in [8]; an announcement of some of our results for the case of a single equation appeared in [3]. To cope with the necessary "groupings" we gave in [8] a definition of $\mu$ slowly decreasing (for $\mu \in E^{\prime}\left(\mathbb{R}^{n}\right)$ ) slightly different from the usual one [21]. Instead of giving the formal definition, let us just mention a few examples of such distributions $\mu$ :

(a) For $n=1$, the definition is the same as before, i.e. condition (9).

(b) Every difference-differential operator $\mu$ (in $n$ variables) is slowly decreasing.

(c) If $\mu$ is a difference-differential operator, $\nu \in E^{\prime}\left(\mathbb{R}^{\mathrm{n}}\right)$ and supp $\nu \subseteq \frac{0}{\operatorname{cv}(\operatorname{supp} \mu)}$, then $\mu+\nu$ is slowly decreasing. Here $\operatorname{cv}($ supp $\mu$ ) denotes the convex hull of the support of $\mu$. Furthermore, there is a number $m$ (depending on $\mu$ ) such that if $\nu \in C^{m}\left(\mathbb{R}^{n}\right)$ and supp $\nu \subseteq \operatorname{cv}(\operatorname{supp} \mu)$, then $\mu+\nu$ is slowly decreasing.

(d) If $\mu$ is of the form (40) it is always slowly decreasing. In this case $\hat{\mu}$ is a distinguished pseudopolynomial,

$$
\hat{\mu}(z)=z_{n}^{m}+\hat{\mu}_{1}\left(z^{\prime}\right) z_{n}^{m-1}+\cdots+\hat{\mu}_{m}\left(z^{\prime}\right),
$$


$z^{\prime}=\left(z_{1}, \ldots, z_{n-1}\right)$ and $\mu_{1}, \ldots, \mu_{m} \in E^{\prime}\left(\mathbb{R}^{n-1}\right)$. Note that after an affine change of coordinates a non-zero polynomial becomes a distinguished pseudo-polynomial.

(e) In the analytic case, every $\mu \in A^{\prime}\left(c^{n}\right), \mu \neq 0$, is slowly decreasing. One can then prove

THEOREM 7 [8]: Let $\mu$ be slowly decreasing, $\quad v=\left\{z \in c^{n}: \hat{\mu}(z)=0\right\}$. There exists a locally finite family of closed subsets $v_{j}$ of $v$, differential operators $\partial_{j}$ and a partition of the index family $J=\{j\}$ into finite subsets $J_{k}$ such that if $\mu * \check{\mathrm{f}}=0$, then

$$
f(x)=\sum_{k}\left(\sum_{j \in J_{k}} \int_{V_{j}} \partial_{j} e^{-i x \cdot z} d \nu_{j}(z)\right)
$$

where the series converges in $E$ and $d \nu_{j}$ are Radon measures with support in $v_{j} \cdot$

REMARK 3: Both Theorems 6 and 7 contain also information about the mass of the measures $d v_{j}$ which corresponds to the estimates (15) of Theorem 4.

As mentioned at the end of Section 4, it is also possible to eliminate the condition that $\mu$ is slowly decreasing.

THEOREM 8 [8]: Let $\mu \neq 0$ be a distribution in $E^{\prime}$ then, with the same notation as in Theorem 7 , we have

$$
\left.f(x)=\lim _{\varepsilon \rightarrow 0} \sum_{k} \sum_{j \in J_{k}} \int_{v_{j}} \partial_{j} e^{-i x \cdot z} d v_{j, \varepsilon}(z)\right),
$$

where the $d \nu_{j, \varepsilon}$ are Radon measures with support in $v_{j}$ and both the series and the limit are taken in the sense of $E$.

For overdetermined systems, we must restrict ourselves to systems for which the Spectral Analysis Theorem holds. This is done by defining what we mean by 
jointly slowly decreasing distributions $\mu_{1}, \ldots, \mu_{\mathbf{r}}$. Once this is done, Theorem 7 holds with $v=\left\{z \in c^{n}: \hat{\mu}_{1}(z)=\cdots=\hat{\mu}_{r}(z)=0\right\}$. The definition itself is quite technical; and the only point worth emphasizing here is that it requires $V$ to be a complete intersection variety of codimension $r$. Thus our result is not strong enough to cover the general situation studied by Ehrenpreis and Palamodov in the case of differential operators (see $[23,59]$ ); on the other hand, we are dealing with analytic varieties instead of algebraic varieties. We refer the reader to [8] for the exact details and pertinent examples; here let us simply observe that one of the main ingredients in the proof is the construction of a function, the Jacobi interpolation function, which plays the role of the Newton polynomial Q from (13). This Jacobi interpolation function was so named because it appears implicitly in [39]. For other insights into the contents of [39] we suggest [30]. Here is an application of our result about systems; other examples appear in [8].

THEOREM 9: Let $S$ denote the unit square in the plane with vertices $A=$ $(0,0), \quad B=(1,0), \quad C=(0,1)$ and $D=(1,1)$. Let $g_{1}, g_{2} \in L^{1}(s)$ be extended as zero outside $S$. Assume further that the eight complex numbers $a_{1}, a_{2}, \ldots, d_{1}, d_{2}$ satisfy the condition

$$
\Delta(\mathrm{A}, \mathrm{B}) \Delta(\mathrm{A}, \mathrm{C}) \Delta(\mathrm{C}, \mathrm{D}) \Delta(\mathrm{B}, \mathrm{D}) \neq 0,
$$

where, for instance,

$$
\Delta(A, B)=\left|\begin{array}{ll}
a_{1} & b_{1} \\
a_{2} & b_{2}
\end{array}\right|=a_{1} b_{2}-a_{2} b_{1} .
$$

Define $\mu_{1}, \mu_{2} \in E^{\prime}\left(\mathbb{R}^{2}\right)$ by

$$
\mu_{j}=a_{j} \delta_{A}+b_{j} \delta_{B}+c_{j} \delta_{C}+d_{j} \delta_{D}+g_{j}, \quad j=1,2 .
$$


If $f \in E\left(\mathbb{R}^{2}\right)$ satisfies the system of equations $\mu_{1} * \check{\mathrm{f}}=\mu_{2} * \not{f}=0$, then $\mathbf{f}$ admits a series representation which converges in $E$ and is of one of the following two kinds,

(i) $f(x)=\sum_{\alpha \in J} c_{\alpha}(x) e^{-i \alpha \cdot x}+\sum_{\alpha \in V \backslash J} c_{\alpha} e^{-i \alpha \cdot x}$, where $\mathbf{v}=\left\{\alpha \in \mathbf{c}^{2}: \hat{\mu}_{1}(\alpha)=\hat{\mu}_{2}(\alpha)=0\right\}, \quad J$ is a finite subset of $\mathrm{V}$ (independent of $f$ ) and the polynomials $c_{\alpha}(x)(\alpha \in J)$ have degree less than the multiplicity of $a$. (It is implicit that $v$ is a discrete set and that for $a \in V \backslash J, c_{\alpha} \in$ C.)

(ii) With $\mathrm{V}$ and $\mathrm{J}$ as above, $\mathrm{V} \backslash \mathrm{J}=\mathrm{v}_{1} \cup \mathrm{v}_{2}, \mathrm{v}_{1} \cap \mathrm{v}_{2}=\emptyset$ and $\mathrm{v}_{2}$ is the disjoint union of the doublets $\{\alpha, \beta\} \quad(\alpha \neq \beta)$, and $f(x)=\sum_{\alpha \in J} c_{\alpha}(x) e^{-i \alpha \cdot x}+\sum_{\alpha \in V_{1}} c_{\alpha}(x) e^{-i \alpha \cdot x}+\sum_{\{\alpha, \beta\} \in V_{2}}\left(c_{\alpha} e^{-i \alpha \cdot x}+c_{\beta} e^{-i \beta \cdot x}\right)$, where the $c_{\alpha}(x)$ in the first sum have the same meaning as in (i) and those with $a \in v_{1}$ are polynomials of degree $\leq 1$.

The two cases arise according to whether the system of algebraic equations

$$
\left\{\begin{array}{l}
a_{1}+b_{1} \xi+c_{1} \eta+d_{1} \xi \eta=0 \\
a_{2}+b_{2} \xi+c_{2} \eta+d_{2} \xi \eta=0
\end{array}\right.
$$

has a pair of simple roots in $c^{2}$ or a single double root.

The proof consists simply of checking that $\mu_{1}, \mu_{2}$ are jointly slowly decreasing and applying the general theory from [8]. Delsarte had proved a weaker version of this theorem in [16] under the additional assumption that the roots of $\mathrm{V}$ were all simple, while in [56] Meyer gave an $\mathrm{L}^{2}$ version of this theorem. A similar expansion holds when the square is replaced by a convex polygon, which improves on a recent result of Yger [74]. 
As a final observation in this section we note that while a Fourier representation for a general class of systems of convolution equations was proposed in [23, Chapter 9], this representation uses a full neighborhood of the variety $\mathrm{V}=$ $\left\{z \in \mathbf{c}^{\mathbf{n}}: \hat{\mu}_{1}(z)=\cdots=\hat{\mu}_{r}(z)=0\right\}$ (even for the case $r=1$ ) and hence is substantially weaker than our results. (The case $r=1$ is very easy and was done in [2].)

6. The equivalent to Theorem 5 is conspicuously missing from the previous section; the reason is that we have only partial results in this direction. Typical examples are the following (see [8]).

THEOREM 10: Let $\mu$ be a difference-differential operator of the form (4) such that the discriminant of the pseudopolynomial $\hat{\mu}$ (see (41)) does not vanish identically. Then if $\mu *$ $=0$ we have

$$
f(x)=\int_{V} e^{-i x \cdot z} d \nu(z)
$$

for some measure $d \nu$ concentrated on $V$, the integral being convergent in $E$.

This theorem is a sharpening of the one proved in [2; 4, Chapter 4] where under the same hypothesis only a representation of the form (38) was obtained. Observe that the second part of Theorem 6 is a particular case of this theorem. Theorem 10 can be extended to distributions $\mu$ for which $\hat{\mu}$ is a distinguished polynomial whose discriminant satisfies certain lower bounds (see [8, 4]).

THEOREM 11: Let $\mu \in E^{\prime}$ be such that at every point of $V=\left\{z \in \mathbf{c}^{\mathrm{n}}\right.$ : $\hat{\mu}(z)=0\}$, we have

$$
|\operatorname{grad} \hat{\mu}(z)| \geq \varepsilon \frac{\exp (-A|\operatorname{Im} z|)}{(1+|x|)^{A}}
$$

for some constants $\varepsilon$, A > 0 . Then the conclusion of Theorem 10 holds. Even under the assumption that $V$ is a manifold (as in Theorem 11) 
we don't know the necessary conditions for a representation of the form (39). This representation corresponds to the situation in Theorem 5 where all the multiplicities are one. The problem of finding necessary and sufficient conditions on slowly decreasing $\mu$ in order that the conclusion of Theorem 10 holds or, more generally, for either representation (38) or (39) from Theorem 6 to hold, is one of the most interesting open questions left in the theory of mean-periodic functions of several variables. Among the very few cases for which we know the answer, is the case of discrete varieties (see [8]); another is the following.

THEOREM 12: Let $\mu \in \mathrm{A}^{\prime}\left(\mathbf{c}^{2}\right), \hat{\mu}$ the distinguished pseudopolynomial

$$
\hat{\mu}(z)=\left(z_{1}-\varphi\left(z_{2}\right)\right)\left(z_{1}-\psi\left(z_{2}\right)\right),
$$

such that $\Delta\left(z_{2}\right)=\varphi\left(z_{2}\right)-\psi\left(z_{2}\right) \neq 0$. Then the conclusion of Theorem 10 holds if and only if the map

$$
\mathbf{F} \longrightarrow \Delta \mathrm{F}
$$

has a closed range when considered as a map from the space

$$
\left\{F \text { entire: }\left|F\left(z_{2}\right)\right| \leq A \exp \left(B\left(\mid \psi\left(z_{2}|+| z_{2} \mid\right)\right) \text {, for some } A, B>0\right\}\right.
$$

into itself.

Summarizing, the general theory of mean-periodic functions of several variables stands now, where the one variable theory stood in the sixties. Still missing is work of the caliber of $[23,59]$ which explored the applications of Theorem 6 to the theory of partial differential equations. Only one chapter of [4, Chapter 3] deals with an application of these theorems to convolution equations (see also a sharpening of these results in [6]). We expect that further developments will come through new results in interpolation theory, in applications to other areas like those in [29] and in the study of general classes for which the 
Spectral Analysis Theorem hold, especially in relation to the study of distributions in symmetric spaces or Lie groups (see [9]).

As S. Bochner has argued in [11, p. 65-66] surveys are usually biased in the sense that in trying to present the gist of the ideas of our predecessors one does not always do justice to their work. He goes on to say that nevertheless this approach has "a very good effect on the whole." Though we cannot expect to escape this general indictment, our hope is that the present work will contribute to further developments in the area of mean-periodicity. That alone should be counted as a positive effect.

ACKNOWLEDGMENT: It is a pleasure to acknowledge support received from the National Science Foundation under Grants MCS 78-00811 and MCS 76-06727 during the preparation of this manuscript. We are also grateful to Professor L. Zalcman for helping us to improve on the readability of the present paper; of course, we alone are to blame for any shortcomings.

\section{REFERENCES}

1. Bellman, R. and Cooke, K., Differential-Difference Equations, Academic Press, 1963.

2. Berenstein, C. A., Convolution operators and related quasianalytic classes, Ph.D. thesis, New York University, 1970.

3. Berenstein, C. A., Interpolation and Fourier analysis in several complex variables, to appear in the Proc. of the Intern. Conf. on Harmonic Analysis and Several Complex Variables, Cortona, Italy, $197 \overline{6}$

4. Berenstein, C. A. and Dostal, M. A., Analytically uniform spaces and their applications to convolution equations, Springer-Verlag, 1972.

5. Berenstein, C.A. and Dostal, M. A., A lower estimate for exponential sums, Bul1. Amer. Math. Soc. 80 (1974), 687-692.

6. Berenstein, C. A. and Lesmes, J., The Cauchy problem for convolution operators. Uniqueness, to appear in Michigan Math. J.

7. Berenstein, C. A. and Taylor, B. A., A new look at interpolation theory for entire functions of one complex variable, to appear in Advances In Math.

8. Berenstein, C. A. and Taylor, B. A., Interpolation problems in $\mathbf{c}^{\mathbf{n}}$ with applications to harmonic analysis, to appear in $\mathrm{J}$. Analyse Math. 
9. Berenstein, C. A. and Zalcman, L., The Pompeiu problem in symmetric spaces, preprint.

10. Bernstein, V., Leçons sur les progrès recents de la théorie des séries de Dirichlet, Gauthier-villars, 1933.

11. Bochner, S., The role of mathematics in the rise of science, Princeton University Press, 1966 .

12. Bochner, S., Lectures on Fourier integrals, Princeton University Press, 1959.

13. Bremermann, H., Distributions, complex variables and Fourier transforms, Addison-Wesley, 1965.

14. Carleman, T., L'intégrale de Fourier et questions qui s'y rattachent, Almquist Wiksel1s, 1944.

15. Delsarte, J., Les fonctions moyenne-périodiques, J. Math. Pures et Appl. 14 (1935), 403-453.

16. Delsarte, J., Théorie des fonctions moyenne-périodiques de deux variables, Ann. of Math. $\underline{72}$ (1960), 121-178.

17. Dickson, D. G., Expansions in series of solutions of linear differencedifferential equations and infinite order differential equations with constant coefficients, Mem. Amer. Math. Soc. 23 (1957).

18. Djakov, P. B. and Mitiagin, B.S., The structure of polynomials in the algebra of entire functions, Institute of Mathematics, Polish Academy of Sciences, preprint $\underline{123}$ (1977).

19. Ehrenpreis, L., Mean-periodic functions. Varieties whose annihilator ideals are principal, Amer. J. of Math. $\underline{77}$ (1955), 293-328 and 731-733.

20. Ehrenpreis, L., Theory of infinite derivatives, Amer. J. of Math. 81 (1959), 799-845.

21. Ehrenpreis, L., Solutions of some problems of division, IV, Amer. J. of Math. 82 (1960), 522-588.

22. Ehrenpreis, L., A Fundamental Principle and some applications, Proc. of the Intern. Symp. on Linear Spaces, Jerusalem, 1961.

23. Ehrenpreis, L., Fourier Analysis in Several Complex Variables, WileyInterscience, 1970 .

24. Ehrenpreis, L. and Malliavin, P., Invertible operators and interpolation in AU spaces, J. Math. Pures et App1. 53 (1974), 165-182.

25. Euler, L:, De integratione aequationum differentialium altiorum graduum, Miscellanea Berol. 7 (1743), 193-242.

26. Gelfond, A. 0., Calcul des Différences Finies, Dunod, 1963. 
27. Gramain, F., Solutions indefiniment dérivables et solutions presque-périodiques d'une équation de convolution, preprint.

28. Grattan-Guinness, I., The Development of the Foundations of Mathematical Analysis from Euler to Riemann, MIT Press, 1970.

29. Griffiths, R. A., Function theory of finite order on algebraic varieties, J. Differential Geometry 6 (1972), 285-306 and ibid. 7 (1972), 45-66.

30. Griffiths, P. A., Variations on a Theorem of Abel, Inventiones Math. 35 (1976), $321-390$.

31. Grudzinski, 0.v., Einigue elementare Ungleichungen für Exponentialpolynome, Math. Ann. 221 (1976), 9-34.

32. Gurevich, D. I., Counterexamples to a problem of L. Schwartz, Funct. Anal. App1. 9 (1975), 116-120.

33. Hansen, S., Uniqueness of the Cauchy problem for convolution operators, preprint.

34. Hilb, E., Zur Theorie der linearen funktionalen Differentialgleichungen, Math. Ann. 78 (1917), 137-170.

35. Hörmander, $L$, An Introduction to Complex Analysis in Several Variables, North-Holland, 1976.

36. Hörmander, L., Generators for some rings of analytic functions, Bull. Amer. Math. Soc. 73 (1967), 943-949.

37. Hörmander, L., Convolution equations in convex domains, Inventiones Math. 4 (1968), 306-317.

38. Hórvath, J., Topological Vector Spaces, Addison-Wesley, 1963.

39. Jacobi, K. G. J., Theoremata nova algebraica circa systema duarum aequationum inter duas variables propositorum, Opera 3 , 287-294.

40. Kahane, J-P., Sur quelques problèmes d'unicité et de prolongement, relatifs aux functions approachables par des sommes d'exponentielles, Ann. Inst. Fourier. $5(1953 / 54), 39-130$.

41. Kahane, J-P., Sur les fonctions moyenne-périodiques bornées, Ann. Inst. Fourier, 7 (1957), 293-314.

42. Kahane, J-P., Lectures on mean-periodic functions, Tata Institute, 1957.

43. Kelleher, J. J. and Taylor, B. A., Finitely generated ideals in rings of analytic functions, Math. Ann. 193 (1971), 225-237.

44. Lacroix, S. F., Traité du calcul différentiel et du calcul intégral, Courcier, 1810-19.

45. Langer, R., Fourier series, the genesis and evolution of a theory, Amer. Math Monthly, 54 (1947), supplement. 
46. Langer, R. E., On the zeros of exponential sums and integrals, Bull. Amer. Math. Soc. 37 (1931), 213-239.

47. Leont'ev, A. F., Differential-difference equations, Mat. Sb. (N.S.) 24 (1949), 347-374. (Amer. Math. Soc. Trans1. (1) 4 (1962), 268-299.)

48. Leont'ev, A. F., Short biography and survey of his work on the occasion of his sixtieth birthday, Russian Math. Surveys 32 (1977), 131-144.

49. Malgrange, B., Existence et approximation des solutions des équations aux dérivées partielles et des équations de convolution, Ann. Inst. Fourier 6 $(1955 / 56), 271-355$.

50. Malgrange, B., Sur les équations de convolution, Rend. Sem. Mat. Univ. e Politec. Torino $19(1959 / 60), 19-27$.

51. Mandelbrojt, S., Séries adhérentes, régularisation des suites, applications, Ganthier-Villars, 1952 .

52. Mandelbrojt, S., Dirichlet series. Principles and methods, Reidel, 1972.

53. Martineau, A., Sur les fonctionelles analytiques, J. Analyse Math. 11 (1963), 1-164.

54. Martineau, A., Distributions et valeurs au bord des fonctions holomorphes, Proc. Intern. Summer Course on the Theory of Distributions. Lisbon, 1964.

55. Meyer, Y., Comportement asymptotique des solutions de certaines équations de cenvolution, J. Math. Pures et Appl. 55 (1976), 69-98.

56. Meyer, Y., Remarques sur un théorème de J. Delsarte, Ann. Inst. Fourier $\underline{26}$ (1976), 133-152.

57. Moreno, C., Ph.D. thesis, New York University, 1971.

58. Myshkis, A. D., General theory of differential equations with a retarded argument, Usp. Mat. Nauk. (N.S.) 4 (1949), 99-141. (Amer. Math. Soc. Trans1. (1) $\underline{4}(\overline{1962}), 207-267$,

59. Palamodov, V. P., Linear Differential Operators with Constant Coefficients, Springer-Verlag, 1970 .

60. Polossuchin, 0., Über eine besondere Klasse von differentiale Funktionalgleichungen, Inaugural Dissertation, Zürich, 1910.

61. Schmidt, E., Über eine Klasse linearer funktionaler Differentialgleichungen, Math. Ann. 70 (1911), 499-524.

62. Schürer, F., Über die Funktional-Differentialgleichungen $f^{\prime}(x+1)=a f(x)$, Ber1. Verhand1. säch. Akad. Wiss. Leipzig, Math. - Phys. K1. 64 (1912), 167-236.

63. Schwartz, L., Théorie des distributions, Hermann, 1950. 
64. Schwartz, L., Théorie générale des fonctions moyenne-périodiques, Ann. of Math. 48 (1947), 857-929.

65. Schwartz, L., Étude des sommes d'exponentielles réelles, Hermann, 1943.

66. Schwartz, L., Approximation d'une fonction quelconque par des sommes d'exponentielles imaginaires, Ann. de la $\underline{\text { Fac. }}$ des Sciences de $\underline{1}$ 'Univ. de Toulouse, 1943.

67. Shapiro, H. S., The expansion of mean-periodic functions in series of exponentials, Comm. Pure App1. Math. 11 (1958), 1-21.

68. Titchmarsh, E. C., Introduction to the Theory of Fourier Integrals, Clarendon Press, 1962 .

69. Verblunsky, S., On a class of differential-difference equations, Proc. London Math. Soc. (3) 6 (1956), 355-365.

70. Volterra, V., Leçon sur 1a théorie mathématique de 1a lutte pour $\underline{\text { la }}$ vie, Gauthier-Vil1ars, $19 \overline{31}$.

71. Waldschmidt, M., Nombres Trascendants, Springer-Verlag, 1974 .

72. Wloka, J., Über das Fundamentalprinzip für Differentialdifferenzenoperatoren, preprint (1978).

73. Wright, E. M., The linear difference-differential equation with constant coefficients, Proc. Roy. Soc. Edinburgh, Sect. A, 62 (1949), 387-393.

74. Yger, A., Une généralisation d'un théorème de J. Delsarte, Séminaire d'Analyse Harmonique (1976-77), 53-75, Publ. Math. Orsay.

75. Zeilberger, D., A new proof to Ehrenpreis' semi-local quotient structure theorem, preprint (1977).

76. Zeilberger, D., Solutions of exponential growth to systems of partial differential equations, preprint (1977).

77. Zygmund, A., Trigonometric series, Cambridge University Press, 1959.

78. Zygmund, A., Notes on the history of Fourier series, in Studies in Harmonic Analysis, ed. J. M. Ash, 1976, 1-19. 


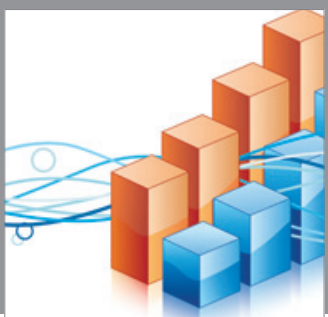

Advances in

Operations Research

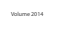

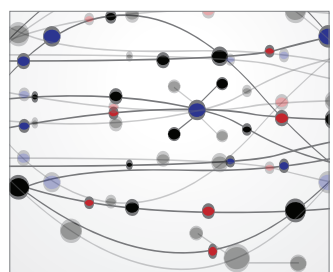

\section{The Scientific} World Journal
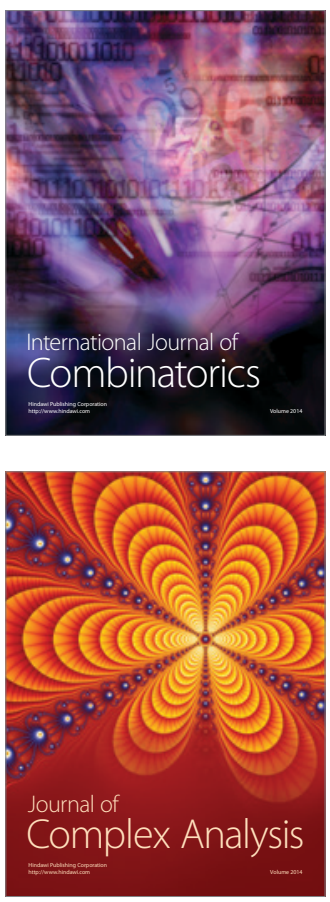

International Journal of

Mathematics and

Mathematical

Sciences
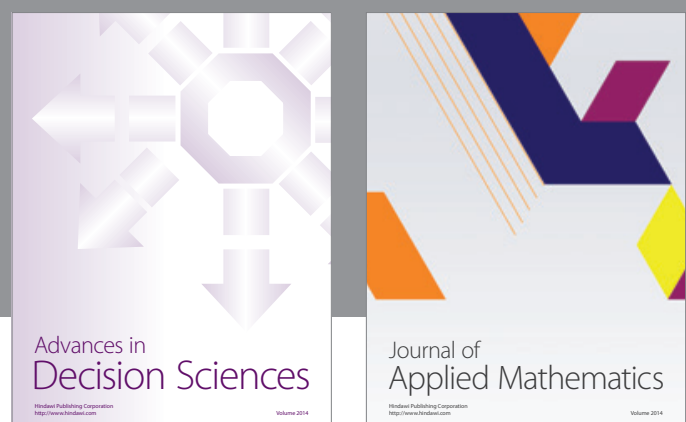

Journal of

Applied Mathematics
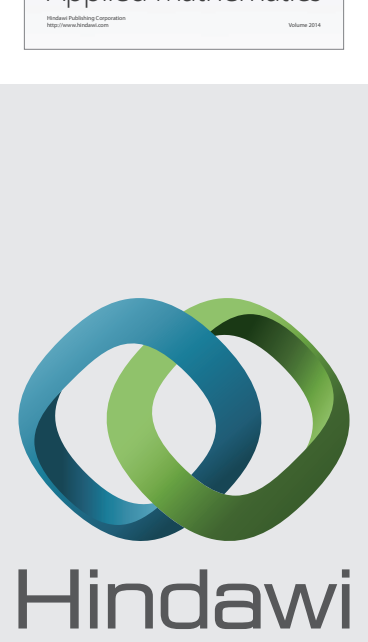

Submit your manuscripts at http://www.hindawi.com
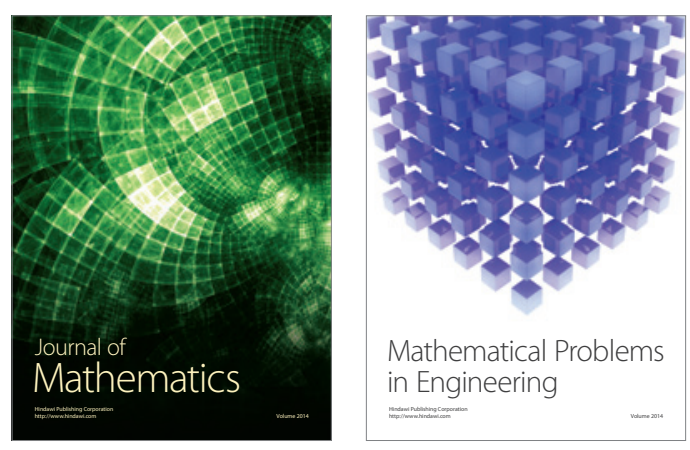

Mathematical Problems in Engineering
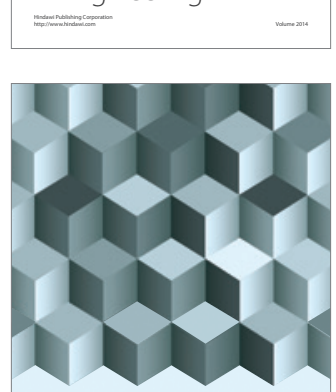

Journal of

Function Spaces
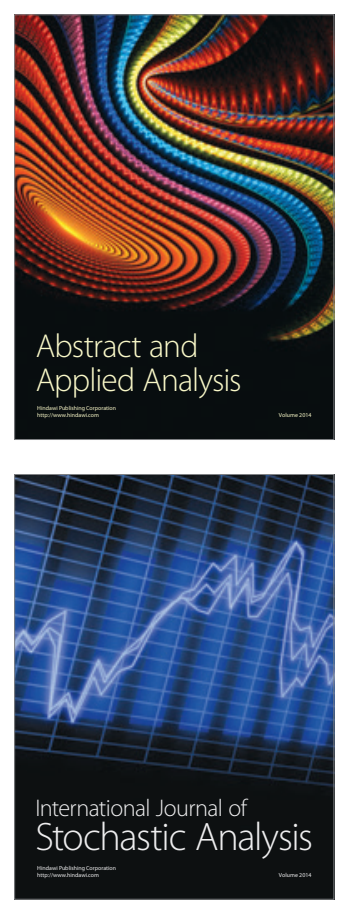

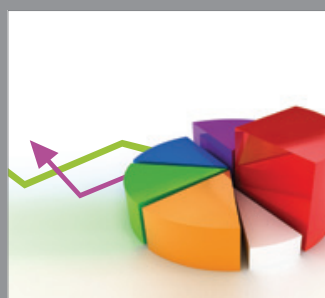

ournal of

Probability and Statistics

Promensencen
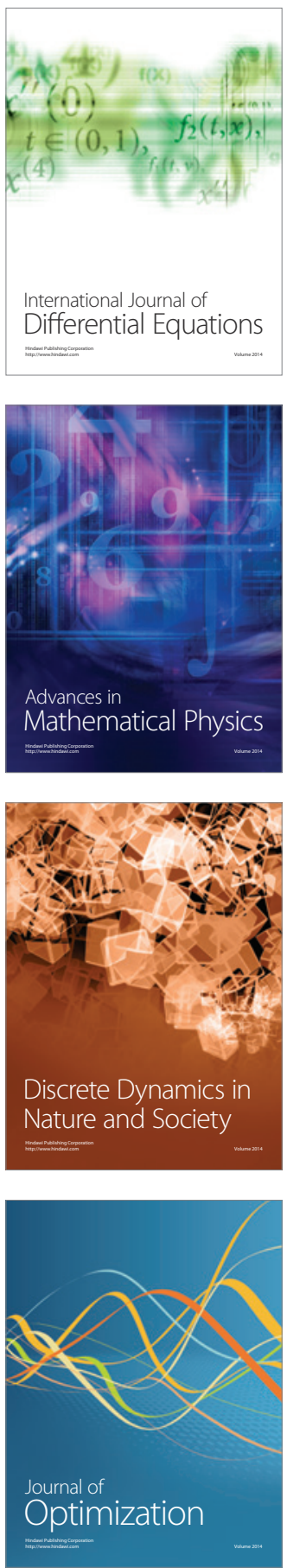